Check for updates

Cite this: Phys. Chem. Chem. Phys. 2021, 23, 18975

Received 31st May 2021,

Accepted 11th August 2021

DOI: $10.1039 / \mathrm{d} 1 \mathrm{cp} 02420$

rsc.li/pccp

\title{
Massive dipoles across the metal-semiconductor cluster interface: towards chemically controlled rectification $\dagger$
}

\author{
Dinesh Bista, (D) Turbasu Sengupta (D) and Shiv N. Khanna (D) *
}

\begin{abstract}
An interface between a metallic cluster $\left(\mathrm{MgAl}_{12}\right)$ and a semiconducting cluster $\left(\mathrm{Re}_{6} \mathrm{Se}_{8}\left(\mathrm{PMe}_{3}\right)_{5}\right)$ is shown to be marked by a massive dipole reminiscent of a dipolar layer leading to a Schottky barrier at metal-semiconductor interfaces. The metallic cluster $\mathrm{MgAl}_{12}$ with a valence electron count of 38 electrons is two electrons short of 40 electrons needed to complete its electronic shells in a superatomic model and is marked by a significant electron affinity of $2.99 \mathrm{eV}$. On the other hand, the metal-chalcogenide semiconducting cluster $\operatorname{Re}_{6} \mathrm{Se}_{8}\left(\mathrm{PMe}_{3}\right)_{5}$, consisting of $\mathrm{Re}_{6} \mathrm{Se}_{8}$ core ligated with five trimethylphosphine ligands, is highly stable in the +2 chargestate owing to its electronic shell closure, and has a low ionization energy of $3.3 \mathrm{eV}$. The composite cluster $\mathrm{Re}_{6} \mathrm{Se}_{8}\left(\mathrm{PMe}_{3}\right)_{5}-\mathrm{MgAl}_{12}$ formed by combining the $\mathrm{MgAl}_{12}$ cluster through the unligated site of $\mathrm{Re}_{6} \mathrm{Se}_{8}\left(\mathrm{PMe}_{3}\right)_{5}$ exhibits a massive dipole moment of $28.38 \mathrm{D}$ resulting from a charge flow from $\mathrm{Re}_{6} \mathrm{Se}_{8}\left(\mathrm{PMe}_{3}\right)_{5}$ to the $\mathrm{MgAl}_{12}$ cluster. The highest occupied molecular orbital (HOMO) of the composite cluster is on the $\mathrm{MgAl}_{12}$ side, which is $0.53 \mathrm{eV}$ below the lowest unoccupied molecular orbital (LUMO) localized on the $\mathrm{Re}_{6} \mathrm{Se}_{8}\left(\mathrm{PMe}_{3}\right)_{5}$ cluster, reminiscent of a Schottky barrier at metal-semiconductor interfaces. Therefore, the combination can act as a rectifier, and an application of a voltage of approximately $4.1 \mathrm{~V}$ via a homogeneous external electric field is needed to overcome the barrier aligning the two states: the $\mathrm{HOMO}$ in $\mathrm{MgAl}_{12}$ with the LUMO in $\mathrm{Re}_{6} \mathrm{Se}_{8}\left(\mathrm{PMe}_{3}\right)_{5}$. Apart from the bias voltage, the barrier can also be reduced by attaching ligands to the metallic cluster, which provides chemical control over rectification. Finally, the fused cluster is shown to be capable of separating electron-hole pairs with minimal recombination, offering the potential for photovoltaic applications.
\end{abstract}

\section{Introduction}

Metal semiconductor junctions play an important role in modern electronics. ${ }^{1-5}$ When a metal is interfaced with a semiconductor, the difference in chemical potentials across the junction causes diffusion of charge carriers that generate an internal dipolar layer and a Schottky barrier. ${ }^{6-14}$ Consequently, an in-built potential accompanied by an internal electric field at the interface results in a directional electrical current under an applied external bias voltage. For bulk interfaces, the nature and quality of the crystal, the diffusion near the interface, and the presence of impurities can all contribute to the alignment of the electronic levels and hence the height of the barrier. ${ }^{15-17}$ As the pace of technology carries us towards smaller length scales, it is interesting to ask if nanoscale motifs exhibiting

Department of Physics, Virginia Commonwealth University, Richmond,

VA, 23284-2000, USA. E-mail: Snkhanna@vcu.edu

$\dagger$ Electronic supplementary information (ESI) available: The optimized structures and relevant data are given in the supplemental information files. All additional data generated or analyzed during this study are available via request to the corresponding author. See DOI: 10.1039/d1cp02420e dipolar characteristics can be designed and if there are novel ways to control the rectifying facets of nano-junctions. An attractive alternative is electronics based on a single molecule ${ }^{18,19}$ composed of two clusters. Such systems exhibit valuable and unusual properties such as current rectification, photovoltaics, electronic switching, and negative differential resistance (NDR). ${ }^{20-31}$ The majority of such research has focused on homoatomic molecular units. ${ }^{32}$

In this work, we investigate the possibilities of connecting a metallic cluster with a semiconducting cluster to test the hypothesis that the charge transfer across the interface might induce significant shifts in the level alignment across the junction. The charge flow could result in a significant dipolar field at the interface leading to a Schottky-like ${ }^{12,13}$ barrier for transmission. The nano-interface could offer rectification characteristics and separate electrons and holes upon the absorption of radiation. The purpose of this work is to investigate these intriguing possibilities by considering a supermolecule composed of an aluminum-based cluster and a metal chalcogenide-based cluster.

The choice of the aluminum-based cluster is based on extensive research over the past three decades that has shown 
that confined nearly free electron gas in metallic clusters exhibits quantum states grouped into shells as in atoms. ${ }^{33-36}$ The levels order as $1 \mathrm{~S}, 1 \mathrm{P}, 1 \mathrm{D}, 2 \mathrm{~S}, 1 \mathrm{~F}, 2 \mathrm{P}$, etc. and clusters with $2,8,18,20,34,40$, etc. valence electrons exhibit closed shells and relative inertness while the clusters with unfilled shells exhibit valence as in atoms. In this work, we have chosen the $\mathrm{MgAl}_{12}$ cluster with 38 valence electrons for the metal-based component. ${ }^{37,38}$ The cluster needs two electrons to acquire a filled shell and is marked by a significant electron affinity enabling it to act as a strong acceptor. Noting this valence state, we chose $\operatorname{Re}_{6} \mathrm{Se}_{8}\left(\mathrm{PMe}_{3}\right)_{5}$ for the semiconductor-based cluster as the previous work ${ }^{39}$ has shown that a $\mathrm{Re}_{6} \mathrm{Se}_{8}$ cluster marked by covalent bonds is highly stable in the +2 chargestate. To facilitate the charge exchange, the $\mathrm{Re}_{6} \mathrm{Se}_{8}$ cluster was decorated with five trimethyl phosphine $\left(\mathrm{PMe}_{3}\right)$ donor ligands. Our previous work has shown that these ligands lead to a lifting of the electronic spectrum that reduces the ionization energy of the cluster without affecting its valence configuration. ${ }^{32,40-42}$ In fact, we will show that it has an ionization energy, ${ }^{43,44}$ which is even lower than that of a Cs atom, making it a suitable donor. Hence, the key idea is to combine semiconductor clusters with lower ionization energy and a stable +2 state with the metallic cluster having higher electron affinity, marked by a stable -2 charge-state. The combination may trigger a significant charge transfer from the semiconducting cluster towards the metallic cluster resulting in a gigantic dipole moment across the junction.

Additionally, the composite cluster might become stable, completing its electronic shell closure. Our key objective is, thus, to examine if the metal-semiconductor junction is marked by a dipolar layer. At small sizes, the role of the valence band edge and the conduction band edge are taken over by the HOMO and LUMO levels. By projecting the projected density of states (PDOS), we examine the HOMO and LUMO of the two individual clusters and check whether the junction across the two clusters presents the analog of the Schottky ${ }^{12,13}$ barrier.

An important parameter characterizing the interface is the height of the Schottky barrier. ${ }^{12,13,15}$ By applying a forward or backward bias voltage, the height of the barrier can be modulated. This allows current flow at low forward bias while the barrier increases for the backward bias. It is interesting to ask if there are other ways of changing the barrier height. Here, we show that at the molecular interface, the height of the barrier can be controlled by attaching ligands. In fact, the barrier can even be inverted by attaching charge transfer ligands offering a chemical approach to modulate the rectification characteristics. Finally, we show that the composite cluster, which mimics Schottky barriers, ${ }^{12,13}$ can effectively separate electron-hole pairs offering the potential for light harvesting.

\section{Results}

\subsection{Electronic properties of the metallic and semiconducting building blocks}

We start with a brief review of the electronic characteristics of the metallic and semiconducting clusters that are building blocks of the composite supermolecule. As mentioned earlier, the quantum confined nearly free electron gas in the metallic cluster can be modeled by a spherical Jellium with a positive background of the size of the cluster. ${ }^{33-36} \mathrm{Al}$ is a trivalent element while $\mathrm{Mg}$ is divalent; the $\mathrm{MgAl}_{12}$ cluster with 38 valence electrons corresponds to $1 \mathrm{~S}^{2} \quad 1 \mathrm{P}^{6} \quad \mathrm{D}^{10} \quad 2 \mathrm{~S}^{2} \quad 1 \mathrm{~F}^{14} \quad 2 \mathrm{P}^{4}$ shells resembling the unfilled $\mathrm{P}$ shell of halogen atoms. As we will show, our first-principles calculations confirm this description. The ground state of the cluster corresponds to a distorted icosahedral structure with an $\mathrm{Mg}$ atom at the center surrounded by $12 \mathrm{Al}$ sites. The $2 \mathrm{P}^{4}$ shell has a filled majority $\mathrm{P}$ shell $\left(2 \mathrm{P}^{3}\right)$, while the minority shell has a single electron resulting in a triplet ground state. Fig. 1(a) shows the calculated adiabatic ionization energy (AIE), adiabatic electron affinity (AEA), dipole moment, and the spin multiplicity $\left(M_{\mathrm{S}}\right)$ of the $\mathrm{MgAl}_{12}$ cluster. The adiabatic energies are obtained by looking at the energy difference between the neutral and ionic clusters in their respective ground states. The relatively high adiabatic ionization energy and electron affinity of the $\mathrm{MgAl}_{12}$ cluster point to its electrophilic character.

The semiconductor counterpart is derived from the ligated metal chalcogenide clusters. We have judiciously chosen the semiconducting cluster as $\mathrm{Re}_{6} \mathrm{Se}_{8}\left(\mathrm{PMe}_{3}\right)_{5}$ as we know from the previous studies ${ }^{32,39}$ that the +2 state of the $\mathrm{Re}_{6} \mathrm{Se}_{8}$ cluster core is highly stable. Furthermore, donor ligands such as tri-ethylphosphine $\left(\mathrm{PEt}_{3}\right)$ or tri-methylphosphine $\left(\mathrm{PMe}_{3}\right)$ can reduce the ionization energy of the cores, making them excellent donors. ${ }^{32,41}$

In experiments, such clusters are generally synthesized with the $\mathrm{PEt}_{3}$ ligands; ${ }^{4,46}$ however, we have chosen analogous $\mathrm{PMe}_{3}$ to reduce the computational cost. We have considered a cluster with five ligands so that one of the unligated metallic sites is open to bind with a counter cluster ( $\mathrm{MgAl}_{12}$ cluster). The semiconducting cluster $\mathrm{Re}_{6} \mathrm{Se}_{8}\left(\mathrm{PMe}_{3}\right)_{5}$ has a triplet ground state with a small HOMO-LUMO (HL) gap of $0.16 \mathrm{eV}$. Fig. 1(d) shows that a larger HOMO-LUMO gap can be realized by removing two electrons. Interestingly, our calculations show that the $\mathrm{Re}_{6} \mathrm{Se}_{8}\left(\mathrm{PMe}_{3}\right)_{5}$ cluster has an ionization energy of $3.32 \mathrm{eV}$, which is lower than that of a Cs atom. ${ }^{43,44}$ This low adiabatic ionization energy (and a small electron affinity of only $0.53 \mathrm{eV}$ ) of the cluster points towards its nucleophilic character. The one-electron energy levels of $\mathrm{MgAl}_{12}$ and $\mathrm{Re}_{6} \mathrm{Se}_{8}(\mathrm{PMe} 3)_{5}$ shown in Fig. 1(c and d) reveal that the energy difference between the HOMO levels of the respective bare clusters is approximately $2.6 \mathrm{eV}$. Furthermore, the transfer of two electrons from HOMO and HOMO-1 levels of the $\mathrm{Re}_{6} \mathrm{Se}_{8}\left(\mathrm{PMe}_{3}\right)_{5}$ cluster to the lowest LUMO-levels of the $\mathrm{MgAl}_{12}$ cluster seems energetically favorable as $\mathrm{MgAl}_{12}$ has two unfilled states in the minority channel. Then the clusters might combine through ionic charge transfer to form a stable composite system with an electronically closed shell.

\subsection{Electronic properties of the $\operatorname{Re}_{6} \mathrm{Se}_{8}\left(\mathrm{PMe}_{3}\right)_{5}-\mathrm{MgAl}_{12}$ dimer}

A composite cluster molecule was formed by combining the $\mathrm{Re}_{6} \mathrm{~S}_{8}\left(\mathrm{PMe}_{3}\right)_{5}$ cluster with the $\mathrm{MgAl}_{12}$ cluster through its unligated site, as shown in Fig. 2(a). Fig. 2(a) also provides the electronic properties and the spin multiplicity of the 


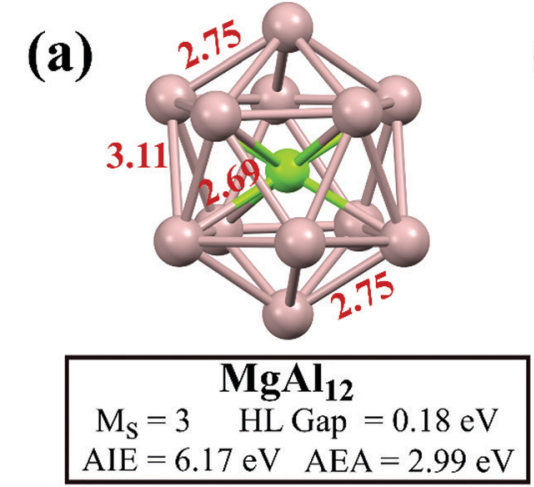

(b)
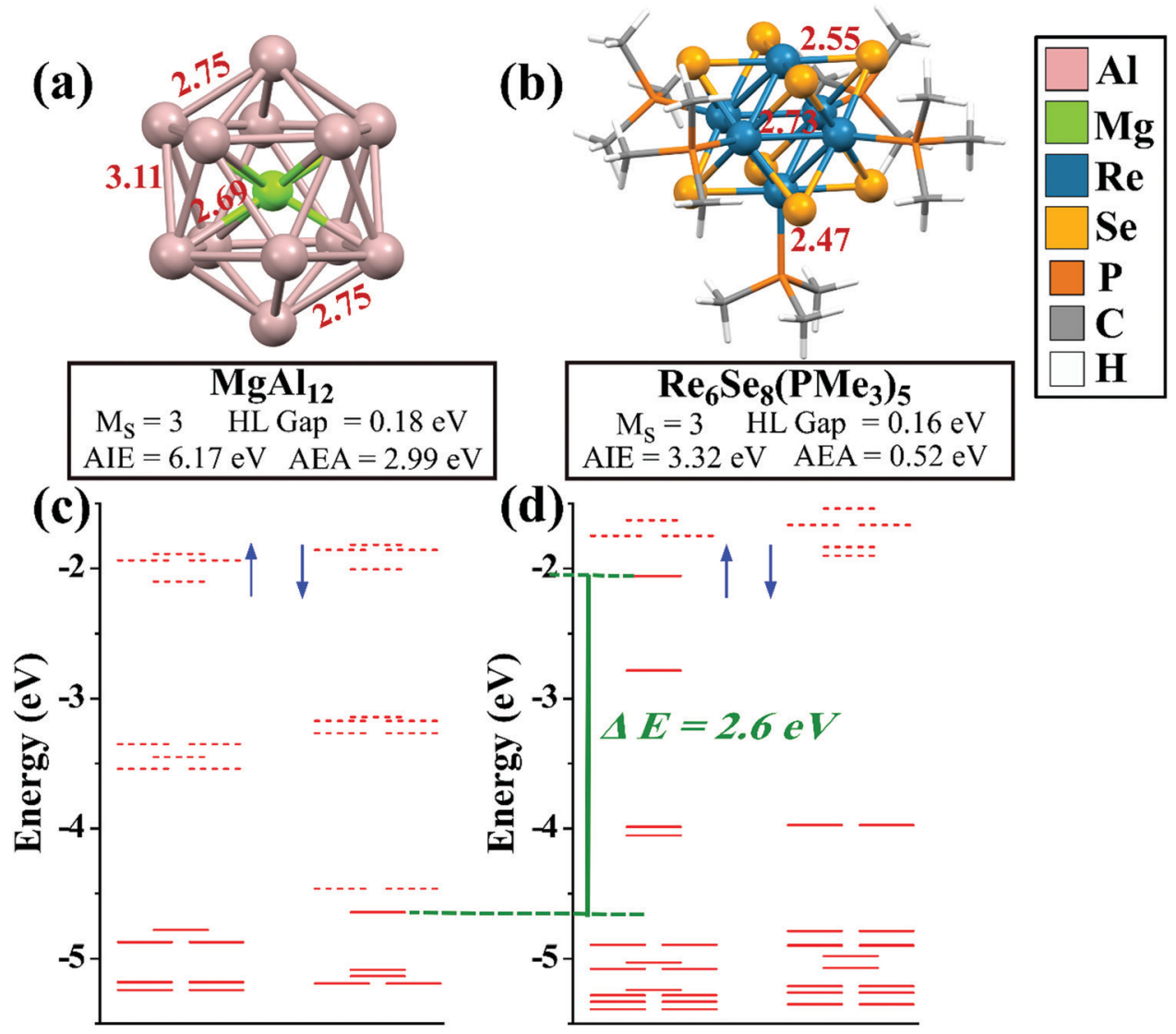

(d) $\mathrm{AIE}=3.32 \mathrm{eV} \quad \mathrm{AEA}=0.52 \mathrm{eV}$

Fig. 1 The ground-state structures and electronic properties of the (a) $\mathrm{MgAl}_{12}$ cluster and (b) $\mathrm{Re}_{6} \mathrm{Se}_{8}\left(\mathrm{PMe}_{3}\right)_{5}$ cluster. (c) One-electron energy levels of $\mathrm{MgAl}_{12}$ and (d) one-electron energy levels of $\mathrm{Re}_{6} \mathrm{Se}_{8}\left(\mathrm{PMe}_{3}\right)_{5}$. The red solid and dash lines represent the occupied and unoccupied levels, respectively. The energy given in green gives the energy difference between the $\mathrm{HOMO}$ levels in $\mathrm{Re}_{6} \mathrm{Se}_{8}\left(\mathrm{PMe}_{3}\right)_{5}$ and $\mathrm{MgAl}_{12}$ clusters. Few bond lengths shown with red text are in $\AA$.

composite cluster. Note that while both bare clusters are triplets, the fused cluster has a singlet ground state with a significantly larger HL gap of $0.53 \mathrm{eV}$ (shown in Fig. 2(b)), more prominent than that of individual bare clusters. We calculated the binding energy (BE) of the molecule using the following expression.

$\mathrm{BE}=E\left(\mathrm{Re}_{6} \mathrm{Se}_{8}\left(\mathrm{PMe}_{3}\right)_{5}\right)+E\left(\mathrm{MgAl}_{12}\right)-E\left(\mathrm{Re}_{6} \mathrm{Se}_{8}\left(\mathrm{PMe}_{3}\right)_{5}-\mathrm{MgAl}_{12}\right)$

Where $E$ is the total energy of the respective systems. The BE is found to be $3.26 \mathrm{eV}$, characteristic of a strong bond. To further characterize the bonding, we calculated the binding energy of an isolated Re-Al since the two clusters are bound with a similar Re-Al bond. The calculated binding energy is $1.51 \mathrm{eV}$. A comparison between the BE of the isolated Re-Al and the composite cluster shows that the bond in the cluster molecule is considerably stronger, suggesting a possible ionic rearrangement. Before we address this, we would also like to add that we calculated the energy required to remove a ligand from the fully ligated $\mathrm{Re}_{6} \mathrm{Se}_{8}\left(\mathrm{PMe}_{3}\right)_{6}$ cluster to create the binding site for combining the $\mathrm{MgAl}_{12}$ cluster. The ligand $\left(\mathrm{PMe}_{3}\right)$ removal energy to attain $\mathrm{Re}_{6} \mathrm{Se}_{8}\left(\mathrm{PMe}_{3}\right)_{5}$ from $\mathrm{Re}_{6} \mathrm{Se}_{8}\left(\mathrm{PMe}_{3}\right)_{6}$ is found to be only $0.88 \mathrm{~V}$, far smaller than the molecular binding energy, showing that the fused cluster molecule is stable.

We calculated the molecule's dipole moment to explore if the strong binding in the composite molecule is due to charge rearrangements. The cluster is marked by a massive dipole moment of 28.38 D. This indicates a substantial charge rearrangement in the molecule. For comparison, we can consider an ionic molecule $\mathrm{NaCl}$, which has a dipole moment of $\sim 9.0$ D. $^{47}$ Furthermore, we carried out a Hirshfeld charge ${ }^{48}$ analysis of the total charge in the two fragments: $\mathrm{MgAl}_{12}$ and $\mathrm{Re}_{6} \mathrm{Se}_{8}\left(\mathrm{PMe}_{3}\right)_{5}$. The results indicate that the $\mathrm{MgAl}_{12}$ fragment gains almost $0.82 \mathrm{e}^{-}$from the metal-chalcogenide cluster during the formation of the fused-molecule, approximately matching with the natural charges ${ }^{49}$ as shown in the ESI $\dagger$ Table S1. The substantial charge rearrangement can be better visualized from a Hirshfeld charge ${ }^{48}$ difference $\left(\Delta q_{\mathrm{H}}\right)$ diagram which shows the amount of charge donated/accepted by individual atoms during the formation of the composite system. Fig. 3a shows the $\Delta q_{\mathrm{H}}$ diagram in which the overall charge difference range is represented by a red-white-blue gradient colormap. A negative (red) color indicates those atoms that have accepted the charge, whereas the positive (blue) value categorizes the donor atoms. The intensity of the color is proportional to the amount of charge accepted or donated by 


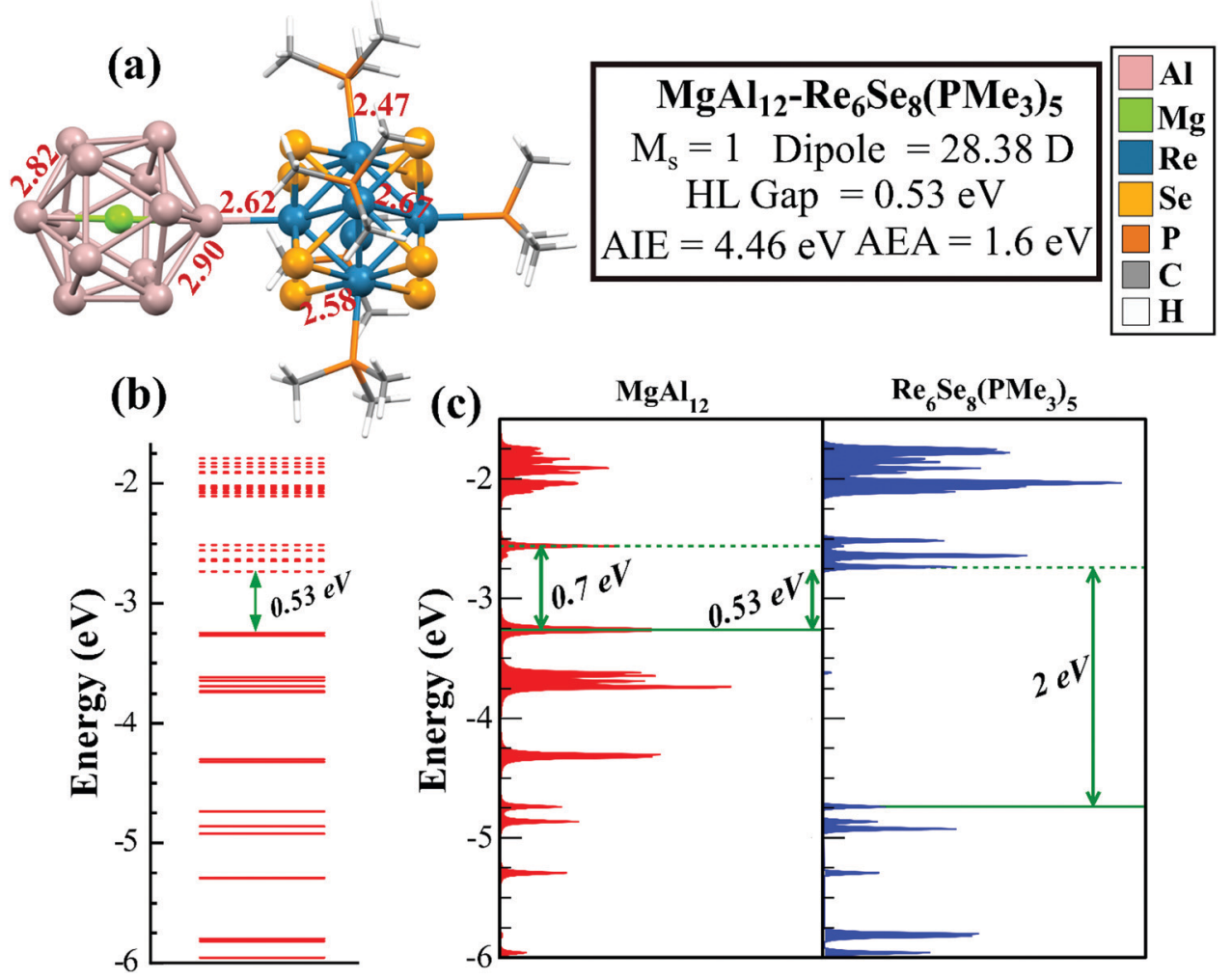

Fig. 2 (a) Ground-state structure and electronic properties of the fused $\mathrm{MgAl}_{12}-\mathrm{Re}_{6} \mathrm{Se}_{8}\left(\mathrm{PMe}_{3}\right)_{5}$. (b) One-electron energy levels of the fused molecule. (c) Projected density of states (PDOS) on $\mathrm{MgAl}_{12}$ and $\mathrm{Re}_{6} \mathrm{Se}_{8}\left(\mathrm{PMe}_{3}\right)_{5}$ fragments of the fused cluster. The green solid and dashed lines represent the $\mathrm{HOMO}$ and LUMO levels in the individual cluster fragments. Few bond lengths shown with red text are in $\AA$.

the respective atoms. It is evident from Fig. 3a that the majority of the $\mathrm{Al}$ atoms in the $\mathrm{MgAl}_{12}$ cluster act as strong electron acceptors during the dimer formation while the Se and Re atoms in the $\mathrm{Re}_{6} \mathrm{Se}_{8}\left(\mathrm{PMe}_{3}\right)_{5}$ fragments act as donors. The four Se atoms near the inter-cluster junction are the strongest donor among the rest. The electron density difference (EDD) of isosurfaces (Fig. 3b) leads to a similar conclusion. During the dimer formation, the electron densities are mainly accumulated on the $\mathrm{Al}$ atoms (red regions) in the $\mathrm{MgAl}_{12}$ fragment, whereas the electron densities are depleted (blue regions) from the $\mathrm{Re}_{6} \mathrm{Se}_{8}\left(\mathrm{PMe}_{3}\right)_{5}$ cluster, especially from the Se atoms that are near the junction.
Charge rearrangements at bulk metal-semiconductor interfaces are inspired by matching the Fermi energy on the metal and semiconductor interfaces. Consequently, we investigated the electronic states in the molecule to obtain insight into the dipole formation. Fig. 2(b) shows the one-electron levels in the molecule, which has a HOMO-LUMO gap of $0.53 \mathrm{eV}$, higher than the corresponding gaps in the individual species, suggesting that the electronic levels in the respective clusters are undergoing substantial rearrangement. To probe these, we investigated the projected density of states (PDOS) of the two fragments in the composite cluster. The continuous and the dotted lines represent

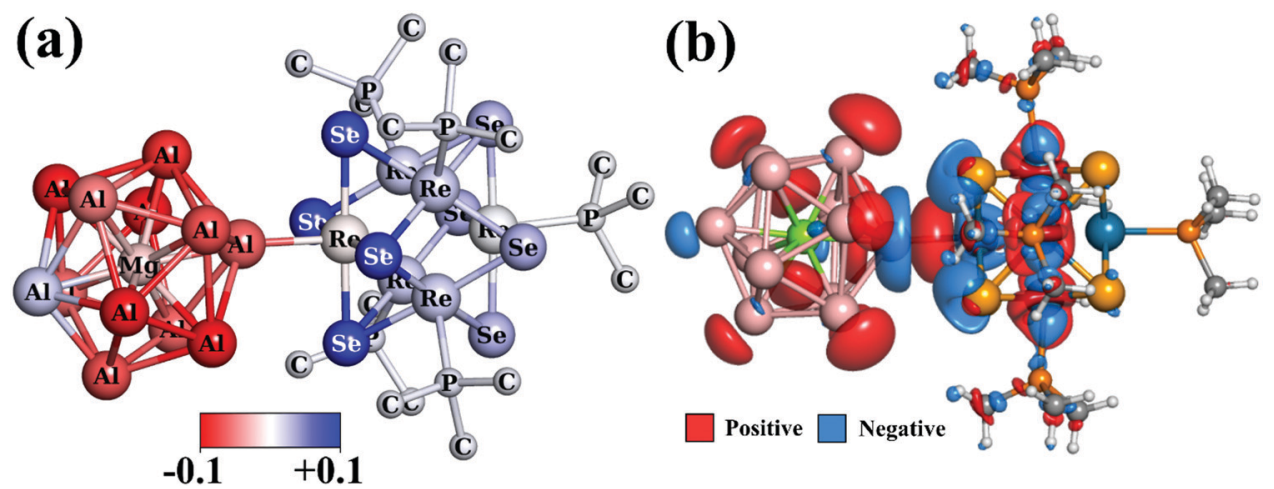

Fig. 3 (a) The Hirshfeld charge difference $\left(\Delta q_{\mathrm{H}}, \mathrm{H}\right.$ atoms are hidden for clarity) and (b) electron density difference (EDD) plot of the fused $M g \mathrm{Al}_{12}-$ $\mathrm{Re}_{6} \mathrm{Se}_{8}\left(\mathrm{PMe}_{3}\right)_{5}$. The red color indicates charge accumulation, and the blue color shows charge depletion during the dimer formation. 
the HOMO and LUMO in the respective cluster-based peaks in PDOS, as shown in Fig. 2(c). The PDOS reveals that the HOMO of the composite cluster molecule is mainly composed of the electronic states located on the $\mathrm{MgAl}_{12}$ cluster, indicative of a lifting of the spectrum on the $\mathrm{MgAl}_{12}$ side.

Furthermore, it is implied that the $\mathrm{MgAl}_{12}$ cluster is receiving the electronic charge, and the two unfilled states above the HOMO in the bare $\mathrm{MgAl}_{12}$ cluster (as shown in Fig. 1(c)) are now filled. By the same token, the LUMO of the composite cluster, mainly consisting of electronic states on the $\mathrm{Re}_{6} \mathrm{Se}_{8}\left(\mathrm{PMe}_{3}\right)_{5}$ side, suggests that the HOMO and HOMO-1 of the majority spin states in the bare $\mathrm{Re}_{6} \mathrm{Se}_{8}\left(\mathrm{PMe}_{3}\right)_{5}$ (as shown in Fig. 1(d)) are getting unoccupied. This results in an apparent large gap of around $2 \mathrm{eV}$, as shown in Fig. 2(c). Such arrangements of the HOMO and LUMO levels in separate regions of the composite cluster are helpful as the states on the individual side can be manipulated using ligands or the external electric field. A clear manifestation of the charge rearrangement is evident from the gigantic electric dipole moment of 28.38 D. To further gain insight into the large dipole moment, we calculated the dipole moment assuming the charge transfer between the centers of mass of two fragments: $\operatorname{Re}_{6} \mathrm{Se}_{8}\left(\mathrm{PMe}_{3}\right)_{5}$ and $\mathrm{MgAl}_{12}$. The approximate distance $\left(r_{\mathrm{e}}\right)$ between their center of masses is $7.224 \AA$, while the charge transfer $(\Delta q)$ is almost $0.82 \mathrm{e}^{-}$. Using the equation dipole $=r_{\mathrm{e}} \cdot \Delta q$, we found an approximate value of $28.45 \mathrm{D}$ compared with 28.38 D found in DFT calculations. The representative figure and calculation of the dipole moment are provided in ESI $\dagger$ Fig. S1.

\subsection{Alignment of barriers using electric field and chemical bias}

The difference in the HOMO-level located on the $\mathrm{MgAl}_{12}$ side and the LUMO on the $\mathrm{Re}_{6} \mathrm{Se}_{8}\left(\mathrm{PMe}_{3}\right)_{5}$ side is $0.53 \mathrm{eV}$, which is indicative of a Schottky-like ${ }^{12,13}$ barrier for the transport of electrons. We wanted to examine if the barrier could be reduced by applying an external homogeneous electric field perpendicular to this interface. To this effect, we applied an external electric field of various strengths to see the positioning of the electronic states. We defined the positive $z$-axis as the axis from the center of mass of the fused cluster towards the $\mathrm{Re}_{6} \mathrm{Se}_{8}\left(\mathrm{PMe}_{3}\right)_{5}$ cluster perpendicular to the junction, as shown in Fig. 4(a). An applied field along the negative $z$-axis, i.e., from the center of mass of the fused cluster towards the $\mathrm{MgAl}_{12}$ cluster, increases the electronic levels in the $\mathrm{MgAl}_{12}$ cluster. At the same time, the metal-chalcogenide counterpart is changed only slightly, as shown in Fig. 4(c and d). Finally, as shown in Fig. 4(d), a field of 0.005 atomic unit, which roughly corresponds to a voltage of $4.1 \mathrm{~V}$, almost aligns the two states: the HOMO level in the $\mathrm{MgAl}_{12}$ region to the LUMO on the $\mathrm{Re}_{6} \mathrm{Se}_{8}\left(\mathrm{PMe}_{3}\right)_{5}$ part.

Reducing the barrier by applying an electric field is similar to the case of solid interfaces; meanwhile, we also examined if there is a chemical way to minimize the barrier. As we have previously shown, the electronic states in the clusters can be shifted by adding ligands. ${ }^{50}$ The donor ligands raise the electronic spectrum while the acceptor ligands move the electronic levels towards lower energies. Here, we wanted to raise the levels in the $\mathrm{MgAl}_{12}$ cluster to match its HOMO with the LUMO on the $\mathrm{Re}_{6} \mathrm{Se}_{8}\left(\mathrm{PMe}_{3}\right)_{5}$ side. Hence, we used donor ligand $\mathrm{N}$-ethyl-2pyrrolidone ( $\mathrm{EP}=\mathrm{C}_{6} \mathrm{H}_{11} \mathrm{NO}$ ), which primarily shifts the electronic spectrum in the upward direction without changing the effective valence count of the system. The EP ligand is structurally similar to the monomeric unit of poly(N-vinyl-2-pyrrolidone) or PVP, which was successfully utilized by Tsunomaya et al. to stabilize gold clusters by charge exchange for catalytic applications. ${ }^{51,52}$

To examine this possibility, we explored the different possible Al-sites on the $\mathrm{MgAl}_{12}$ cluster to attach the EP ligands. We observed that the attachment of EP ligands lowers the gap

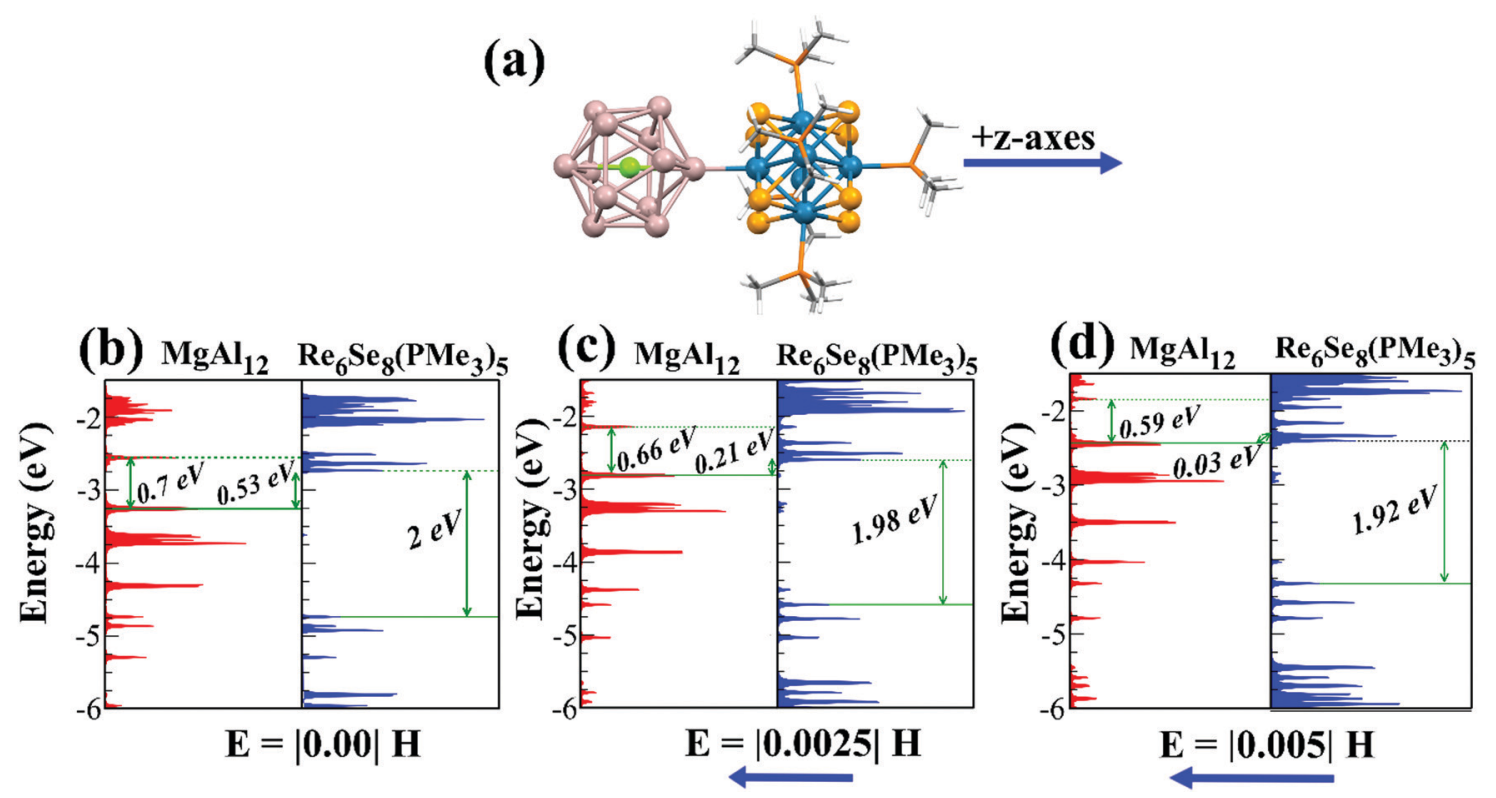

Fig. 4 (a) The $+z$-axis for the applied electric field. (b) PDOS for $E=0.00$ Hartree (H), (c) LDOS for $E=-0.0025 \mathrm{H}$ and (d) PDOS for $E=-0.005 \mathrm{H}$. 


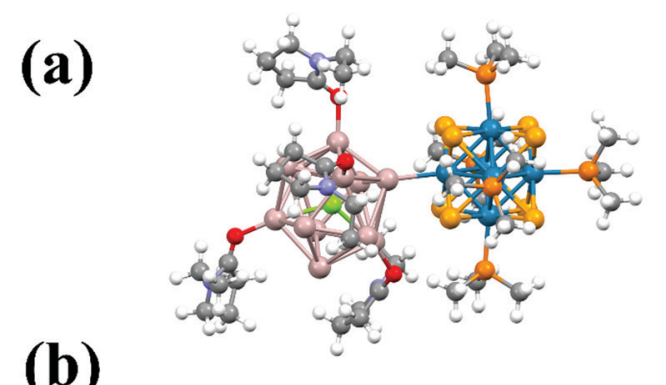

(b)

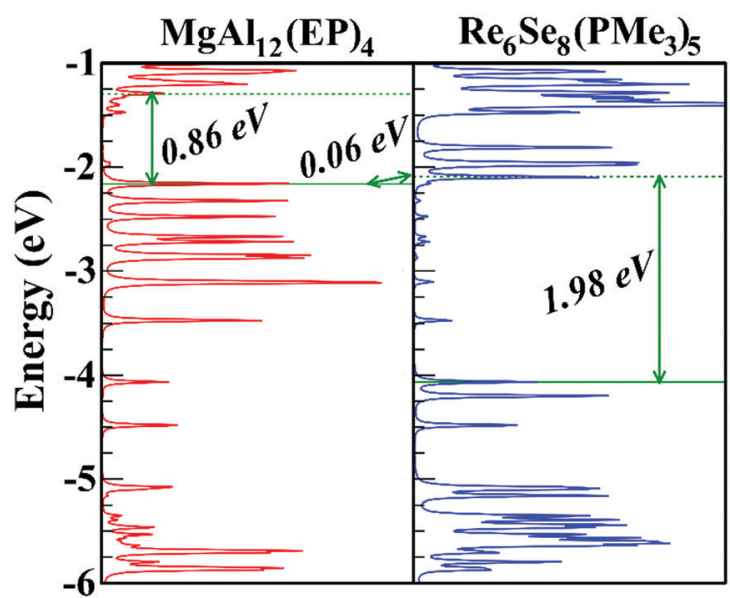

Fig. 5 (a) Ground state structure of the $\mathrm{MgAl}_{12}(\mathrm{EP})_{4} \mathrm{Re}_{6} \mathrm{Se}_{8}\left(\mathrm{PMe}_{3}\right)_{5}$ cluster (b) PDOS in fragments: ligated metallic clusters $\mathrm{MgAl}_{12}(\mathrm{EP})_{4}$ and $\mathrm{Re}_{6} \mathrm{Se}_{8}\left(\mathrm{PMe}_{3}\right)_{5}$. The green solid and dashed lines represent the HOMO and LUMO levels in the individual fragments.

by raising the electronic levels of the $\mathrm{MgAl}_{12}$ cluster with only a marginal influence on the electronic states on the $\mathrm{Re}_{6} \mathrm{Se}_{8}\left(\mathrm{Pme}_{3}\right)_{5}$ side. Finally, attaching $4 \mathrm{EP}$ ligands to the $\mathrm{MgAl}_{12}$ cluster almost aligns the HOMO of the $\mathrm{MgAl}_{12}$ with the LUMO of the $\mathrm{Re}_{6} \mathrm{Se}_{8}\left(\mathrm{PMe}_{3}\right)_{5}$ side. Fig. 5(a) shows the ground state structure of the $\mathrm{MgAl}_{12}(\mathrm{EP})_{4} \mathrm{Re}_{6} \mathrm{Se}_{8}\left(\mathrm{PMe}_{3}\right)_{5}$ cluster among all possible isomers with $4 \mathrm{EP}$ ligands attached to the Al-sites. The effective gap is reduced to only $0.06 \mathrm{eV}$, as shown by the PDOS plot in Fig. 5(b). Changing the barriers by adding ligands opens a new area where one can use chemical and electrical means to control the barrier heights in these nanodevices.

\subsection{Optical absorption and electron hole separation}

We now examine the potential application of the composite molecule in photovoltaics to separate the electron-hole pairs with marginal recombination. The gigantic dipole moment of 28.38 D in the fused molecule suggests that the charge could be separated. To examine this possibility, we analyzed the location of the HOMO in the anionic and cationic clusters. Fig. 6(a) shows that the spin densities of the anionic and cationic composite clusters are localized entirely on the separate regions. The molecule's absorption spectrum using timedependent density functional theory is shown in Fig. 6(b), which shows that the first significant optical excitation energy is found at approximately $1.0 \mathrm{eV}$. This excitation energy is significantly higher than the HL gap of $0.53 \mathrm{eV}$ in the system,

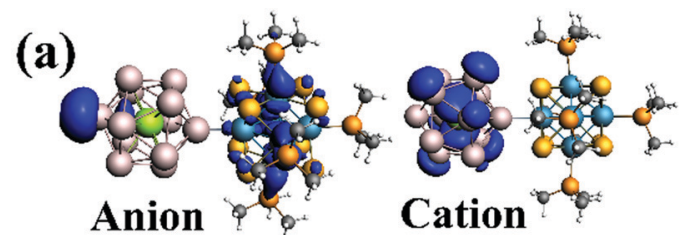

(b)

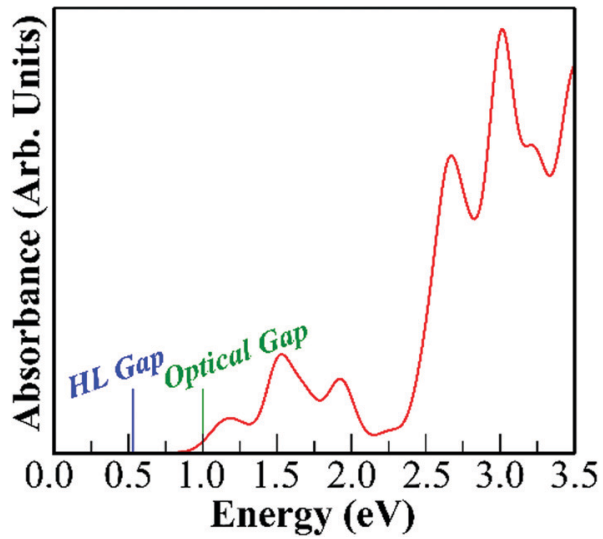

Fig. 6 (a) The spin density on the $\mathrm{MgAl}_{12}-\mathrm{Re}_{6} \mathrm{Se}_{8}\left(\mathrm{PMe}_{3}\right)_{5}$ cluster shows the location of the electrons and holes (b) the optical absorption spectra of the composite $\mathrm{MgAl}_{12}-\mathrm{Re}_{6} \mathrm{Se}_{8}\left(\mathrm{PMe}_{3}\right)_{5}$ cluster.

showing that the electron-hole recombination is unlikely as one relaxes to the states near the HOMO-LUMO gap. The lowest energy excitation is found around $0.53 \mathrm{eV}$; however, the associated oscillator strength is significantly small, with a mere value of $4.125 \times 10^{-05}$ atomic unit. Furthermore, the electrons and holes of the lowest energy excitations are localized on different clusters, as shown in ESI $\dagger$ Fig. S2. These excitations are optically very weak, leading to a low probability of electron-hole recombination via dipole allowed processes. Hence, such a Schottky barrier ${ }^{12,13}$ type superatomic molecule provides an attractive way to create separated electron-hole pairs for light harvesting.

\section{Conclusions}

We have investigated a fused molecule consisting of the metallic and the semiconducting cluster that exhibits a Schottky-like $e^{12,13}$ characteristic at its interface. It is known that a forward bias opens the switch for the current flow in the bulk systems. The molecular Schottky barrier ${ }^{12,13}$ also shows a similar phenomenon. When the external homogeneous electric field is applied to the composite cluster molecule, the electronic levels on the metallic cluster $\mathrm{MgAl}_{12}$ are shifted with respect to the electronic states in the semiconductor cluster $\mathrm{Re}_{6} \mathrm{Se}_{8}\left(\mathrm{PMe}_{3}\right)_{5}$, thereby aligning the electronic levels at the interfacial region. Hence, the molecule opens the possibility of favored electron transport through the junction at a lower forward bias. Furthermore, a novel strategy to manipulate the electronic states in the fused molecule using the ligands, labeled as chemical bias, is also proposed. In the studied system, the EP ligands attached to the metallic cluster serve the purpose of alignment of 
electronic levels on the individual cluster to open a path for favored electron transport. Finally, the composite molecule with the gigantic dipole moment is shown to separate electron-hole pairs with negligible recombination, making it an attractive candidate for photovoltaic applications.

\section{Methods}

\subsection{Theoretical techniques}

Theoretical studies in this work are based on first-principles density functional methods using the Amsterdam Density Functional (ADF) program. ${ }^{53}$ The exchange-correlation functional proposed by Perdew, Burke, and Ernzerhof (PBE), which uses the generalized gradient approximation (GGA) was used. ${ }^{54}$ The TZ2P basis set ${ }^{55,56}$ was used with a large frozen electron core. The local minimum for each structure was found using the quasi-Newton ${ }^{57}$ method with no symmetry restriction ascertaining the lowest energy structures. The threshold for the electronic convergence was set to $1 \times 10^{-8}$ Hartree, and the default convergence criteria (as implemented in $\mathrm{ADF}^{53}$ ) were utilized for all the geometric optimizations. We have probed several spin multiplicities ranging from 1 to 5 to determine the most energetically stable ground state structures for the anions, neutral, and cation clusters (see Table S2 in the ESI†).

Moreover, relativistic effects were incorporated using the zero-order regular approximation (ZORA). ${ }^{58}$ Natural population analysis (NPA) was performed by using the NBO $6.0^{49}$ module implemented within ADF. ${ }^{53}$ The electric field calculations were performed by aligning an external electric field with the cluster along the $z$-axis. The positive $z$-axis is defined as the axis from the center of mass of the composite cluster towards the $\mathrm{Re}_{6} \mathrm{Se}_{8}\left(\mathrm{PMe}_{3}\right)_{5}$ cluster. The voltage was computed by multiplying the applied homogeneous electric field with the distance between the terminal atoms along the $z$-axis of the composite cluster. Finally, the TD-DFT $^{59}$ calculation was employed to calculate the optical absorptions, with the lowest 500 dipole-allowed excitations mimicking the optical spectra.

\section{Author contributions}

D. B. and T. S. conducted all the calculations. S. N. K., T. S. and D. B. analyzed the results and the manuscript was written by S. N. K., D. B. and T. S.

\section{Conflicts of interest}

The authors declare no competing interest.

\section{Acknowledgements}

The authors acknowledge support by a grant from the US Department of Energy (DOE) under the award DE-SC0006420.

\section{References}

1 S. Hong, Y. Yoon and J. Guo, Appl. Phys. Lett., 2008, 92, 083107.

2 Y. Liu, P. Stradins and S.-H. Wei, Sci. Adv., 2016, 2, e1600069.

3 O. Yaffe, L. Scheres, S. R. Puniredd, N. Stein, A. Biller, R. H. Lavan, H. Shpaisman, H. Zuilhof, H. Haick and D. Cahen, Nano Lett., 2009, 9, 2390-2394.

4 S. G. Louie and M. L. Cohen, Phys. Rev. B: Solid State, 1976, 13, 2461.

5 H. R. Banjade, J. Pan and Q. Yan, Phys. Rev. Mater., 2021, 5, 014005.

6 W. Shockley and H. J. Queisser, J. Appl. Phys., 1961, 32, 510-519.

7 L. M. Terman, Solid-State Electron., 1962, 5, 285-299.

8 S. J. Jiao, Z. Z. Zhang, Y. M. Lu, D. Z. Shen, B. Yao, J. Y. Zhang, B. H. Li, D. X. Zhao, X. W. Fan and Z. K. Tang, Appl. Phys. Lett., 2006, 88, 031911.

9 N. Yaacobi-Gross, M. Soreni-Harari, M. Zimin, S. Kababya, A. Schmidt and N. Tessler, Nat. Mater., 2011, 10, 974-979.

10 M.-Y. Li, Y. Shi, C.-C. Cheng, L.-S. Lu, Y.-C. Lin, H.-L. Tang, M.-L. Tsai, C.-W. Chu, K.-H. Wei, J.-H. He, W.-H. Chang, K. Suenaga and L.-J. Li, Science, 2015, 349, 524-528.

11 Q. Kong, W. Lee, M. Lai, C. G. Bischak, G. Gao, A. B. Wong, T. Lei, Y. Yu, L.-W. Wang, N. S. Ginsberg and P. Yang, Proc. Natl. Acad. Sci. U. S. A., 2018, 115, 8889-8894.

12 R. T. Tung, Mater. Sci. Eng., R, 2001, 35, 1-138.

13 R. T. Tung, Appl. Phys. Rev., 2014, 1, 011304.

14 B. L. Sharma, Metal-semiconductor Schottky barrier junctions and their applications, Springer Science \& Business Media, 2013.

15 Y.-J. Chang and J. L. Erskine, Phys. Rev. B: Condens. Matter Mater. Phys., 1983, 28, 5766.

16 Y.-W. Lian, Y.-S. Lin, J.-M. Yang, C.-H. Cheng and S. S. Hsu, IEEE Electron Device Lett., 2013, 34, 981-983.

17 J. Tersoff and W. A. Harrison, Phys. Rev. Lett., 1987, 58, 2367. 18 P. Gehring, J. M. Thijssen and H. S. van der Zant, Nat. Rev. Phys., 2019, 1, 381-396.

19 Y. Komoto, S. Fujii, M. Iwane and M. Kiguchi, J. Mater. Chem. C, 2016, 4, 8842-8858.

20 A. Aviram and M. A. Ratner, Chem. Phys. Lett., 1974, 29, 277-283.

21 P. Avouris, Acc. Chem. Res., 2002, 35, 1026-1034.

22 H. B. Akkerman, P. W. M. Blom, D. M. de Leeuw and B. de Boer, Nature, 2006, 441, 69-72.

23 H. He, R. Pandey, J. U. Reveles, S. N. Khanna and S. P. Karna, Appl. Phys. Lett., 2009, 95, 192104.

24 H. He, R. Pandey, G. Mallick and S. P. Karna, J. Phys. Chem. C, 2009, 113, 1575-1579.

25 X. Zhong, R. Pandey, A. R. Rocha and S. P. Karna, J. Phys. Chem. Lett., 2010, 1, 1584-1589.

26 L. Zhu and S. N. Khanna, J. Chem. Phys., 2012, 137, 164311. 27 L. Zhu, M. Qian and S. N. Khanna, J. Chem. Phys., 2013, 139, 064306.

28 B. Fu, M. A. Mosquera, G. C. Schatz, M. A. Ratner and L.-Y. Hsu, Nano Lett., 2018, 18, 5015-5023. 
29 N. Xin, J. Guan, C. Zhou, X. Chen, C. Gu, Y. Li, M. A. Ratner, A. Nitzan, J. F. Stoddart and X. Guo, Nat. Rev. Phys., 2019, 1, 211-230.

30 B. M. Boardman, J. R. Widawsky, Y. S. Park, C. L. Schenck, L. Venkataraman, M. L. Steigerwald and C. Nuckolls, J. Am. Chem. Soc., 2011, 133, 8455-8457.

31 G. Lovat, B. Choi, D. W. Paley, M. L. Steigerwald, L. Venkataraman and X. Roy, Nat. Nanotechnol., 2017, 12, 1050-1054.

32 A. C. Reber, V. Chauhan, D. Bista and S. N. Khanna, Nanoscale, 2020, 12, 4736-4742.

33 W. D. Knight, K. Clemenger, W. A. de Heer, W. A. Saunders, M. Y. Chou and M. L. Cohen, Phys. Rev. Lett., 1984, 52, 2141.

34 S. N. Khanna and A. W. Castleman, Quantum phenomena in clusters and nanostructures, Springer Science \& Business Media, 2013.

35 D. E. Bergeron, A. W. Castleman, T. Morisato and S. N. Khanna, Science, 2004, 304, 84-87.

36 A. C. Reber and S. N. Khanna, Acc. Chem. Res., 2017, 50, 255-263.

37 Z. Luo, C. J. Grover, A. C. Reber, S. N. Khanna and A. W. Castleman Jr, J. Am. Chem. Soc., 2013, 135, 4307-4313.

38 C. J. Grover, A. C. Reber and S. N. Khanna, J. Chem. Phys., 2017, 146, 224301.

39 Z. Zheng, J. R. Long and R. H. Holm, J. Am. Chem. Soc., 1997, 119, 2163-2171.

40 V. Chauhan, A. C. Reber and S. N. Khanna, J. Am. Chem. Soc., 2017, 139, 1871-1877.

41 A. C. Reber, D. Bista, V. Chauhan and S. N. Khanna, J. Phys. Chem. C, 2019, 123, 8983-8989.

42 D. Bista, T. Sengupta, A. C. Reber and S. N. Khanna, J. Phys. Chem. A, 2021, 125, 816-824.

43 A. Kramida, Y. Ralchenko and J. Reader, NIST Atomic Spectra Database (Ver. 5.7.1), National Institute of Standards and Technology, Gaithersburg, MD, USA, 2019.
44 J. Deiglmayr, H. Herburger, H. Saßmannshausen, P. Jansen, H. Schmutz and F. Merkt, Phys. Rev. Appl., 2016, 93, 013424.

45 T. G. Gray and R. H. Holm, Inorg. Chem., 2002, 41, 4211-4216.

46 L. F. Szczepura, M. K. Oh and S. A. Knott, Chem. Commun., 2007, 4617-4619.

47 F. J. Lovas, E. Tiemann, J. S. Coursey, S. A. Kotochigova, J. Chang, K. Olsen and R. A. Dragoset, NIST Diatomic Spectral Database (version 2.1), National Institute of Standards and Technology, Gaithersburg, MD, 2005.

48 F. L. Hirshfeld, Theor. Chim. Acta, 1977, 44, 129-138.

49 E. D. Glendening, C. R. Landis and F. Weinhold, J. Comput. Chem., 2013, 34, 1429-1437.

50 V. Chauhan, A. C. Reber and S. N. Khanna, Nat. Commun., 2018, 9, 1-7.

51 H. Tsunoyama, N. Ichikuni, H. Sakurai and T. Tsukuda, J. Am. Chem. Soc., 2009, 131, 7086-7093.

52 H. Tsunoyama and T. Tsukuda, J. Am. Chem. Soc., 2009, 131, 18216-18217.

53 G. te Velde, F. M. Bickelhaupt, E. J. Baerends, C. Fonseca Guerra, S. J. A. van Gisbergen, J. G. Snijders and T. Ziegler, J. Comput. Chem., 2001, 22, 931-967.

54 J. P. Perdew, K. Burke and M. Ernzerhof, Phys. Rev. Lett., 1996, 77, 3865-3868.

55 E. Van Lenthe and E. J. Baerends, J. Comput. Chem., 2003, 24, 1142-1156.

56 D. P. Chong, E. Van Lenthe, S. Van Gisbergen and E. J. Baerends, J. Comput. Chem., 2004, 25, 1030-1036.

57 L. Fan and T. Ziegler, J. Chem. Phys., 1991, 95, 7401-7408.

58 E. van Lenthe, J. G. Snijders and E. J. Baerends, J. Chem. Phys., 1996, 105, 6505-6516.

59 S. J. A. Van Gisbergen, J. G. Snijders and E. J. Baerends, Comput. Phys. Commun., 1999, 118, 119-138. 